\title{
Implementation Analysis of RETT-Gen Routing Protocol
}

\author{
Ms. Uma K. Thakur \\ III Sem., M.E. [WCC], \\ G.H.R.C.E., Nagpur
}

\author{
Prof. S. A. Chhabria \\ Professor, PG Dept. of CSE, \\ G.H.R.C.E., Nagpur
}

\begin{abstract}
Wireless sensor networks are composed of a large number of sensor nodes with limited energy resources energy efficiency is a key design issue that needs to be enhanced in order to improve the life span of the entire network.

In RETT-gen, we transform the expected lifetime of each sensor node to an equivalent temperature, and then by using the heat dissipation equations, we find the hottest path for ending data to the base station, which will not always be the shortest path.
\end{abstract}

\section{Keywords}

Clustering, Wireless sensor network, Routing protocol, energy efficient.

\section{INTRODUCTION}

A typical sensor node consists mainly of a sensing circuit for signal conditioning and conversion, a digital signal processor, and radio links[6]. During the life cycle of the sensor node, each event or query will be followed by a sensing operation, performing necessary calculations to derive a data packet and send this packet to its destination[1]. Thus, we divide the energy consumption model into the following models; the communication energy consumption model, followed by the computation energy consumption model.

An ad-hoc wireless network of large numbers of such inexpensive but less reliable and accurate sensors can be used in a wide variety of commercial and military applications. These include target tracking, security, environmental monitoring, system control, etc.[2]. Routing protocol of WSNs plays an important role in network organization and data transmitting, which can further affect the life span and reliability of networks [3]. Clustering is one of the important approaches to save energy in order to keep the node life. Most of the previous works have been done about clustering-based routing protocol [7].

\section{Objectives:}

Each sensor node has a fixed number of transmission power levels. An example of such sensor nodes [3]. Each sensor node is equipped with an omnidirectional antenna and able to dynamically adjust its transmission power level depending on the distance over which it transmits a data packet [1]. Energy efficiency is one of the most important design constraints in wireless sensor network architectures [4].

Clustering increase system capacity. Using clustering enables better resource allocation and helps improve power control [11]. Clustering and routing must be efficient in terms of processing complexity and message exchange. Scalability (i.e., a network is extended to a larger size) is essential in the routing algorithm [1].

\section{Operations of the protocol.}

RETT-gen protocol included following steps:

1. Clustering

2. Cluster-head election

3. Network clusters localization

4. Equalization based routing

5. Cluster-head temperature calculation

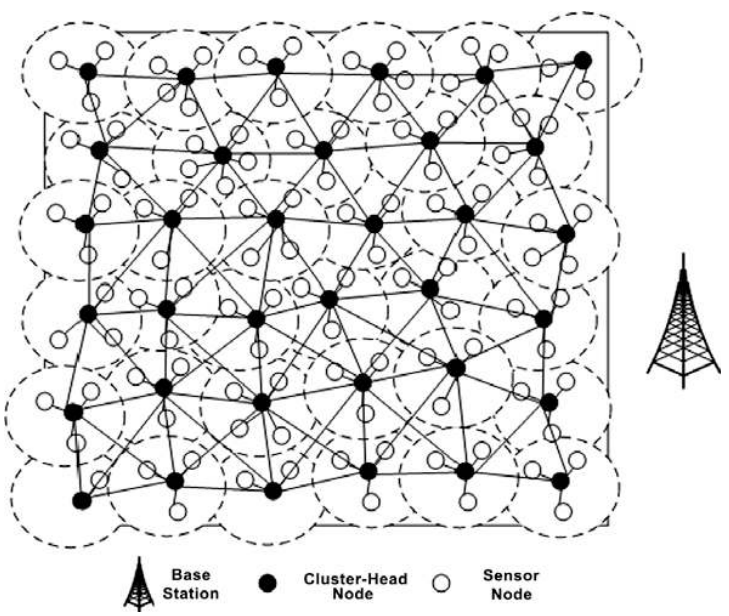

Fig. 1 Localization of cluster-head nodes.

As being a cluster head node is much more energy intensive than being a non-cluster head node, this requires that each node take its turn as cluster head [11].

\subsection{Clustering:}

In clustering process, partitions the network into clusters, each with its own cluster-head node. Each cluster head is responsible 
for processing the data, sending the data and participating in routing decisions [1]. The clustering process independent of the network diameter [3].

\subsection{Cluster head election:}

The role of the cluster heads is to perform routing in the network. This conserves energy since only one sensor node at a time in each cluster is required to constantly operate with an active radio interface. The head of the cluster calculates the average value of the energy for all sensor nodes within the cluster. The head of the cluster divides the average by a scaling variable. When the cluster head's energy reaches the threshold, it queries the sensor nodes in the cluster for their current energy. Then it selects the sensor node with the highest energy to become the new head of the cluster and resorts to being a normal sensing node [3].

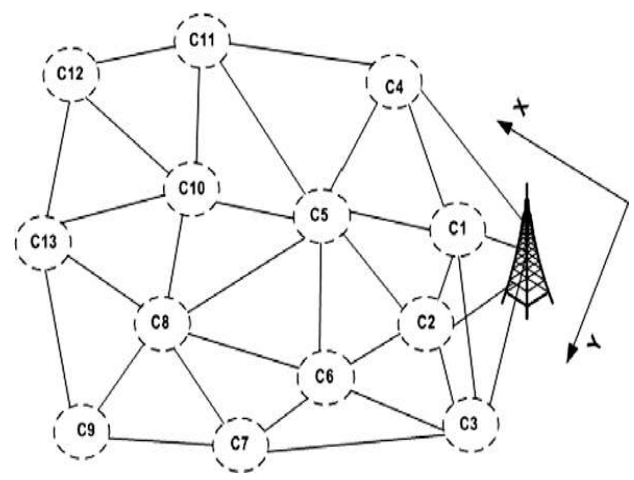

Fig. 2. Neighboring cluster-heads.

\subsection{Network clusters localization:}

To find out the position information for each cluster-head node, RETT-gen uses range measurement between clusters (i.e., clusters-head nodes) to build a network coordinate system. The actual distance estimation via received signal strength is used to obtain the range between two clusters [1]. Performing clustering on a sensor network deployment prior to localization has two advantages. First, it creates a regular pattern (Fig. 1) from which location information can be extracted. Second, it helps reduce the amount of communication overhead since only the cluster-heads need to be involved in the initial phase of the localization [2].

\subsection{Equalization based routing:}

Our proposed protocol is based on the analogy of heat conductivity and its goal is to equalize the energy of the sensor nodes throughout the network so that the majority of the sensor nodes consume their energy at the same relative rate regardless of the physical positions of these nodes. In RETT-gen, we distribute our routing decisions based on the expected energy of clusters in the network. In RETT-gen, we distribute our routing decisions based on the expected energy of clusters in the network.

\subsection{Cluster-head temperature:}

The calculation of the cluster-head temperature is divided into two phases; the first phase determines the direction of the routes between the clusters-heads and the base station (analogous to a constant gravity, pulling the data in the general direction of the base station), and the second phase selects a suitable path or route that the cluster-head (the source) must use to send its data towards the base station on a per-packet and per-hop basis.

\section{CONCLUSION:}

Maximizing the life span of an entire sensor network by means of power equalization.

RETT-gen spreads the energy load among all the sensor nodes. Hence clusters close to the base station as well as the clusters far away survive until the end.

\section{REFERENCES:}

1) Khaled Matrouk Bjorn Landfeldt, RETT-gen: A globally efficient routing protocol for wireless sensor networks by equalising sensor energy and avoiding energy holes, Ad Hoc Networks 7 (2009) Published by Elsevier B.V, 2008

2)S. Bandyopadhyay, E. Coyle, An energy efficient hierarchical clustering algorithm for wireless sensor networks, Proceedings of the 22nd Annual Joint Conference of the IEEE Computer and Communications Societies (INFOCOM) (2003).

3) Jian Chen, Zhihui Yin, Deshi Li, Tao Sun, A Distributed and Effective Cluster Routing Protocol of Sensor Networks, 2008.

4) K. Matrouk, B. Landfeldt, Energy-conservation clustering protocol based on heat conductivity for wireless sensor networks, in: IEEE International Conference on Intelligent Sensors, Sensor Networks and Information Processing (ISSNIP), 2004

5) Wei Bo Hu Han-ying Fu Wen, An Improved LEACH Protocol for Data Gathering and Aggregation in Wireless Sensor Networks , International Conference, 2008

6)R. VIidhyapriya, P.T. Vanathi, Conserving energy in Wireless Sensor Network, MPOT , 2007 7) Ruihua Zhang1, Lin Wang1, Shichao Geng 2 , Zhiping Jia1-A Balanced Cluster Routing Protocol of Wireless Sensor Network, (ICESS)(2008)

8) G. Huang, X. Li Jing He, Energy-Efficiency Analysis of Cluster-Based Routing Protocols in Wireless Sensor Networks, IEEEAC (2006).

9) M. Ye, C. Li, G. Chen, J. Wu, EECS: an energy efficient clustering scheme in wireless sensor networks, in: Proceedings of IEEE International Conference (IPCCC), (2005).

10) Lu Cheng, Depei Qian, Weiguo Wu, An Energy Efficient Weight-clustering Algorithm in Wireless Sensor Networks, JapanChina Joint Workshop,(2008).

11) Wendi B. Heinzelman, Anantha P. chandrakasan, An Application-Specific Protocol Architecture for Wireless Microsensor Networks, IEEE TWC, (2002) 